\title{
Development of a Two Factor Authentication for Vehicle Parking Space Control based on Automatic Number Plate Recognition and Radio Frequency Identification
}

\author{
Friday Chisowa Chazanga ${ }^{1}$, Jackson Phiri ${ }^{2}$ \\ Department of Computer Science \\ The University Zambia \\ Lusaka, Zambia
}

\author{
Sebastian Namukolo ${ }^{3}$ \\ Department of Electrical \& Electronic Engineering \\ The University Zambia \\ Lusaka, Zambia
}

\begin{abstract}
This paper proposed a two factor authentication for vehicle access controls using Automatic Number Plate Recognition (ANPR) and Radio Frequency Identification system (RFID) for the University of Zambia (UNZA) vehicle access points. The University of Zambia is experiencing increasing challenge of car parking space and vehicle access controls to and within campus premises. The survey that was conducted reviewed that members of staff found difficulties finding parking spaces due to intrusion. The survey also reviewed that vehicles have been stolen within campus parking areas without detection. An access control system using integrated ANPR and RFID technologies was developed to provide five authentication states that met different vehicle access point's requirement. It was built with 'ORed' and 'ANDed', logic settings to achieve five different states of authentication levels, each suited for a particular access point. The ANRP system used the vehicle number plate to authenticate the vehicle through the use of the camera. On the other hand, the RFID system used the drivers' card/tag through the RFID card reader to authenticate the user. Daily transaction records were sent to the security center where information would easily be retrieved. Illegal access to restricted areas, threats of theft of motor vehicles and failed transaction recording system was amicably solved by this proposal.
\end{abstract}

Keywords-RFID; ANPR; Vehicle access control; two-factor authentication

\section{INTRODUCTION}

With an ever increasing volume of vehicles that enter and leave the University of Zambia (UNZA) campus premises; monitoring and tracking of vehicles, information retrieval as well as control of vehicles' access using the current manual system has become impractical. Attempts to authenticate every vehicle and driver at various access points by the security personnel leads to congestion and inefficient time management. This study proposed a model that electronically provided authentication to vehicle access into and out of car parks and campus premises. The system also provided monitoring and tracking of vehicle movements through number plate captures and driver identification. The two main objectives of the study was to develop a two factor authentication system modal for vehicle access control based on RFID and ANPR technologies. The implementation was done by using a boom gate barrier system prototype for vehicle access in and out of premises at particular access points. The modal offered five configuration access states that would be tailored to suit access authentication requirement at different access points. The study was necessitated by the outcome of a survey that was carried out that highly recommended for a secure electronic vehicle access control system that would keep records of both vehicle and driver activities. A proposed model addressed these requirements and the limitations and challenges reviewed in the survey by the provision of a two factor authentication with five configurable authentication states.

Studies have been done using technologies such as Number Plate Recognition as well as Radio Frequency Identification vehicle access controls elsewhere. However, no such study has been done here in Zambia towards such local security concerns. In this research, a robust two-factor authentication access control mechanism for vehicles in and out of a restricted premise or car park, using Automatic Number Plate Recognition (ANPR) and Radio Frequency Identification (RFID) was developed. A boom gate barrier system prototype was built for controlling vehicle access in and out of the car park and restricted premises. According to [1][2] a multifactor authentication provides a more secure systems in the Cyber space and other areas of security importance. ANPR is a tool that has the capability to detect and recognize the vehicle's number plate and provide the information regarding it with reference to the data base [3] [4]. On the other hand, (RFID) is an $\mathrm{ADC}$ technology that uses radio-frequency waves to transfer data between a reader and a tag to identify, categorize, and track objects among others. It is fast, reliable and does not require sight of line or contact to communicate [5].

This research outcome can however be fundamentally applied to many institutions where control and security of vehicle movements is strictly adhered to. We however confined our analysis and application to the University of Zambia.

\section{LITERATURE REVIEW}

In this section, we examined Scholar databases to find related literature. The examination reviewed that studies have 
been done on Multifactor Authentication security systems in many areas of Artificial Intelligence, Machine Learning and Neural Networks. Many researchers have also proposed different algorithms covering a wider range of vehicle access control systems using technologies such as RFID and ANPR. Infra-Red sensors have been used in the detection of objects and actuation of the system cameras to photograph an image. In this research, a review has been done on multifactor authentication, RFID and ANPR technologies, and IR sensors.

\section{A. Multifactor Authentication}

A multifactor authentication has been deemed a more secure security implementation in many areas of security concern. In [1][2] a Multifactor Authentication System to create a more secure authentication to minimize cybercrime was developed. The system was employed through a fuser block of an artificial neural network and adaptive neural-fuzzy inference system.

\section{B. Automatic Number Plate Recognition (ANPR)}

Definition: Automatic Number Plate Recognition (ANPR) is a system where a car number plate is recognized and identified automatically. Initially, as the car approaches, the camera is actuated by an infrared lighting to allow it take a photo. The camera senses and takes a picture of the vehicle[6] [7]. The vehicle captured image will be sent to pre-processing stage where Grey Image Conversion takes place. The second stage involves removal of undesirable Lines. Vertical Edge Detection Algorithm are implemented to eradicate undesirable lines and scan the license plate. The Desired Details of the image around the plate area are highlighted and extracted at the third stage[8]. Since ANPR is an image processing technology which uses number plate to identify the vehicle, there is no need for any additional hardware to be installed on vehicles. Fig. 1 shows ANPR image processing flowchart from image input to plate character display.

1) Application: Tracking of vehicles for traffic offences committed is a major concern to law enforcers. Admittance of vehicles to Amkkah in the Pilgrimage seasons is restrictive to specific vehicles on particular days. In order to deny access and track violators of this order, Mohandes proposed an Intelligent System for Vehicle Access Control using RFID and ANPR Technologies where ANPR was used for admittance to the premises and RFID technology was used for tracking [4]. An ant robbery system that granted permission for registered vehicle passage using ANPR was proposed and implemented [10]. A method for the vehicle number plate recognition from the image using a special form of optical character recognition (OCR) to control vehicles in restricted car parks was discussed. It used the optical character recognition to read number plates through CCTV systems, which enables vehicle registration numbers to be stored, analyzed and retrieved, as required[11]. In [12] proposed was an efficient automatic vehicle identification system using the vehicle number plate for various applications including automatic toll tax collection, parking system, Border crossings, Traffic control and stolen cars.

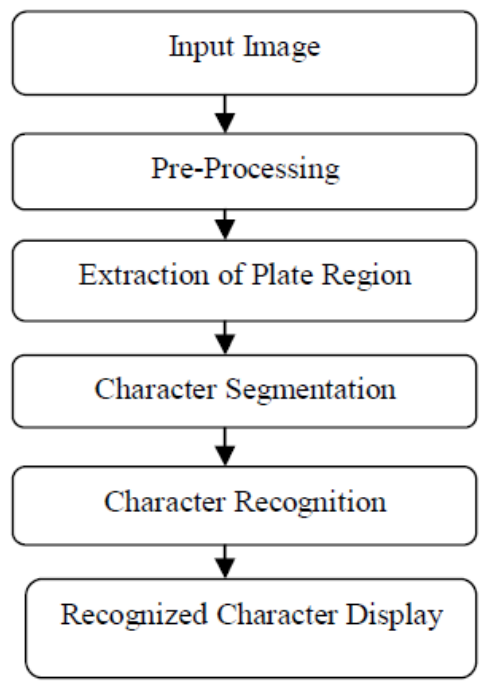

Fig. 1. Image Processing Flowchart[9].

\section{Radio Frequency Identification (RFID)}

1) Definition: Radio Frequency Identification (RFID) technology is a technology that uses radio waves to identify people or objects from a distance. It combines two main components, that is, a tag and a reader. The two components are used for proof of identity purposes[13]. A cypher is stored in the tag which is attached to an object. With this attached tag, objects are uniquely identifiable. The code embedded in the tag is transmitted to the reader wirelessly. In this way, the reader gets information about the object[14].

2) The Structure of RFID system: RFID has a combination of a reader, a tag (transponder) and a data processing system. Data processing unit is the systems backend that stores information such as scanned product descriptions. The data processing system is either a personal computer or microcontroller[15]. It uses electromagnetic coupling to recognize items, animals and humans as distinctive objects. The transceiver (reader) produces radio signals that activates the tag, scans the tag and communicates any data collected to a processing unit through an antenna[16]. Reading of information contained in the tag by the reader is done from a distance without making any physical contact or requiring a line of sight. The read information is sent to the data base for logical decision making, either to permit or deny access. The backend or processing unit comprises of a database and an application interface. When information is received by the backend, it's taken to the database where it is manipulated[17]. Below is a simplified RFID system. Fig. 2 and 3 illustrates RFID system operations. Initially, the RFID reader drives electromagnetic waves to the tags antenna. Current is induced in the tag causing it to reflect to the RFID reader modulated radio frequency signal containing data. The reader sends the data to the middleware for database query. Database response determines the system's logic decision making on the access[14]. 


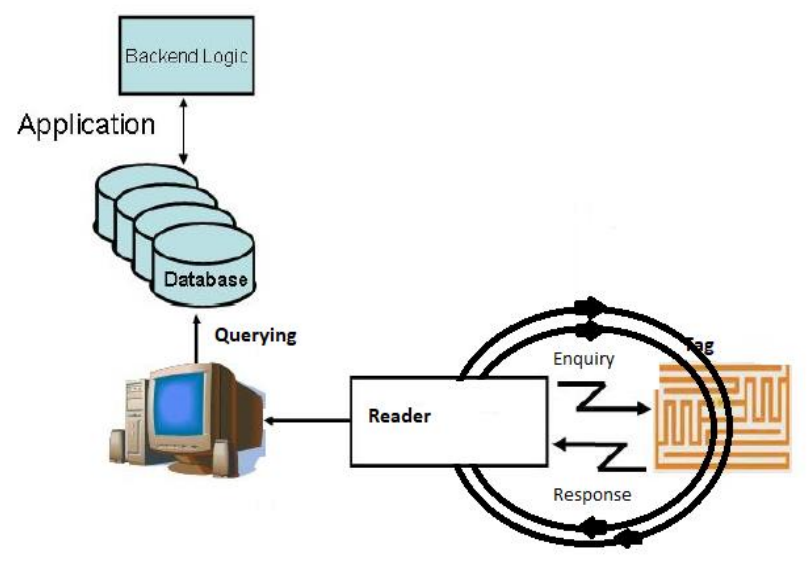

Fig. 2. A Simplified RFID System[14].

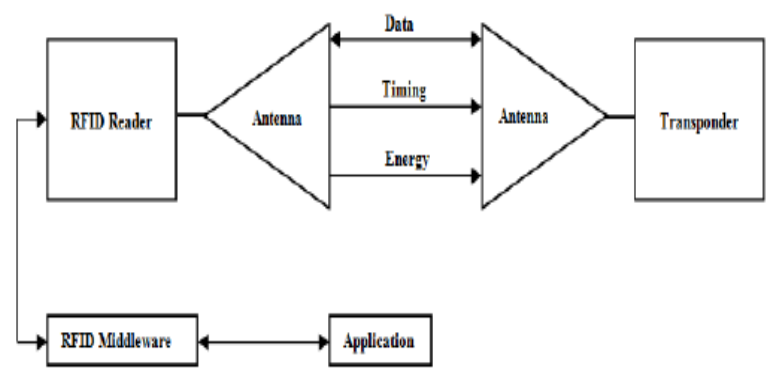

Fig. 3. RFID System Operation[14].

3) RFID Operations: A Radio Frequency Identification RFID system consists of a tag, reader, and middleware software. Tags have a microchip with an antenna coiled internally. The tags are generally categorized as active or passive. Active tag use batteries or power source to power the circuitry and generate broadcasted signals. It propagates electromagnetic waves containing information in the far field to the reader. The tag operates in the UHF and microwaves frequency bands. Because of its dependability on a power source, active tag has a short life span. Its cost is high and the size does not support usage in some applications. This makes an active RFID tag not suitable for regular usage. It however has a longer read range than the passive tags [18][19]. Passive RFID tags are independent of internal power source. They are also called 'pure passive', 'reflective' or 'beam powered'. They get their functional power from the reader through electromagnetic induction in the tag's antenna. The tag then reflects modulated radio frequency signal to the reader. On the other hand a reader is an interrogating device that has internal and often times external antennas that send and receive signals. The middleware software allows the system read/write tags and provides a means to catalogue and query tag information.[19]. Passive RFID have benefits of lengthy lifespan and its small physical size makes it appropriate for useful adhesive label. As such, passive RFID tags are used for many applications[18].
4) Application: Review carried out by [20] indicates that RFID market is rapidly expanding in a number of factors that include; automotive, transportation, logistics, healthcare and military sectors, Military Assets, consumables, conveyances, vehicles sensing Smart and Secure Trade lanes global initiative Intermodal containers, Logistics Items, assets and conveyances. In Passenger transport/automotive vehicle, premises and computer access, and ticketing system. This has brought about expanded number of users and suppliers of RFID on the market. With an ever increasing demand for parking spaces. Effective use of available spaces becomes important. The RFID system was designed and developed to park cars in a multilevel parking area automatically[5]. RFID usage and applications have increased over the years, an outline of its history and growing industrial usage has been highlighted in a research papers titled "the History of RFID" and "The adoption of RFID technology in the retail supply chain," [21][22]. In [23][24] an automatic toll collection using RFID was developed. They used IR sensors to trigger the RFID reader to take readings. Design and implemented a one authentication vehicular access control using RFID. [22] observed that due to the technology's versatility, the device has undergone rigorous exploration by several organization. [21] listed some of the uses to include highway and bridge tolls, livestock tracking, transportation freight tracking and motorcycle manufacturing.

\section{Infra-Red Sensors}

1) Introduction: Infra-Red sensors are extensively used as a presence trigger. Its output however is influenced by factors such as, displacement of the object from the IR sensor, the direction and speed of object travel and the object shape[25]. Infra-Red sensors are classified into two types, photon detectors and thermal detectors based on their principle of operation. In photon detectors the radiation is absorbed within the material by interaction with electrons either bound to lattice atoms or to impurity atoms or with free electrons. The changes in the electronic energy distribution in the atom generates output electrical signals. In thermal detectors the incident radiation is absorbed to change temperature of the material object, the subsequent adjustments in some physical properties of the object generates an electrical output[26].

2) Operations: There is a large variety of IR sources, each used for different purposes[27]. All objects are composed of continually vibrating atoms. Higher energy atoms vibrating more frequently leads to radiate some form of infrared radiation known as thermal radiation due to generated electromagnetic waves. This is what makes Infrared radiation such a powerful resource[28]. It allows for the ability to detect and gather information of an environment without the need of visible light[29]. The technologies reviewed above will be used in a two factor authentication system [30] based on RFID and ANPR in order to improve on the security levels of the proposed system. 


\section{MethodOLOGY}

- Materials

Materials use for the project include include; a boom gate, IR sensors, cameras, stepper motor, computer, database, software, LCD display, contactors, relays, electronic components, tags, reader microcontroller/Arduino board and cables, survey tools.

\section{- Baseline Study}

A baseline survey was conducted at the University of Zambia Great East Road Main Campus to ascertain the need for an Electronic Vehicle Access Security Control System. The main areas under focus were the three main entrance and exit points to and from the University campus premises. The three included the Great East Road Main Entrance, the Kamloops entrance and the Lufwanyama entrance. The other areas of focus were the Members of staff car parking areas within the University premises that included, School of Education Staff Car park servicing members of staff in the School of education, Administration Car park servicing the top management staff, School of Engineering car park servicing members of staff from the School of Natural Sciences, School of Humanities, and School of Engineering and the transport Yard. The areas were carefully selected because they by university regulations have entry and exit points for selected classes of vehicles.
Three categories of respondents were selected. These are Security Personnel, Members of Staff and Students/Visitors. The first category of respondents involved all the available security personnel on the campus on the particular day. Forty members of staff and forty students/visitors were selected.

\section{- Current Vehicle Access Control System}

The current vehicle access controls into the university campus and through to the car parking areas is manually done. Members of staff of the university are issued with identity cards for general use. Those with vehicles are also issued with vehicle stickers that are displayed on the vehicle windscreens. Upon reaching the entrance or exit point, the security personnel requests to see the University of Zambia car sticker or member of staff identity card. When either of the two is produced, the vehicle will be allowed to gain access to the premises failure to which the vehicle is returned. Guards use a book to take details of the transaction such as recording the vehicle number plate and time of entry and exit into and out of the premises.

The current manual system reviewed a lot of challenges and inefficiencies causing vehicle congestion and time wastage during peak hours. Tracing vehicle activities and stolen cars have also been very difficult because security personnel are not able to inspect each vehicle that needs access.

Fig. 4 defines the existing process of vehicle authentication using the manual system.

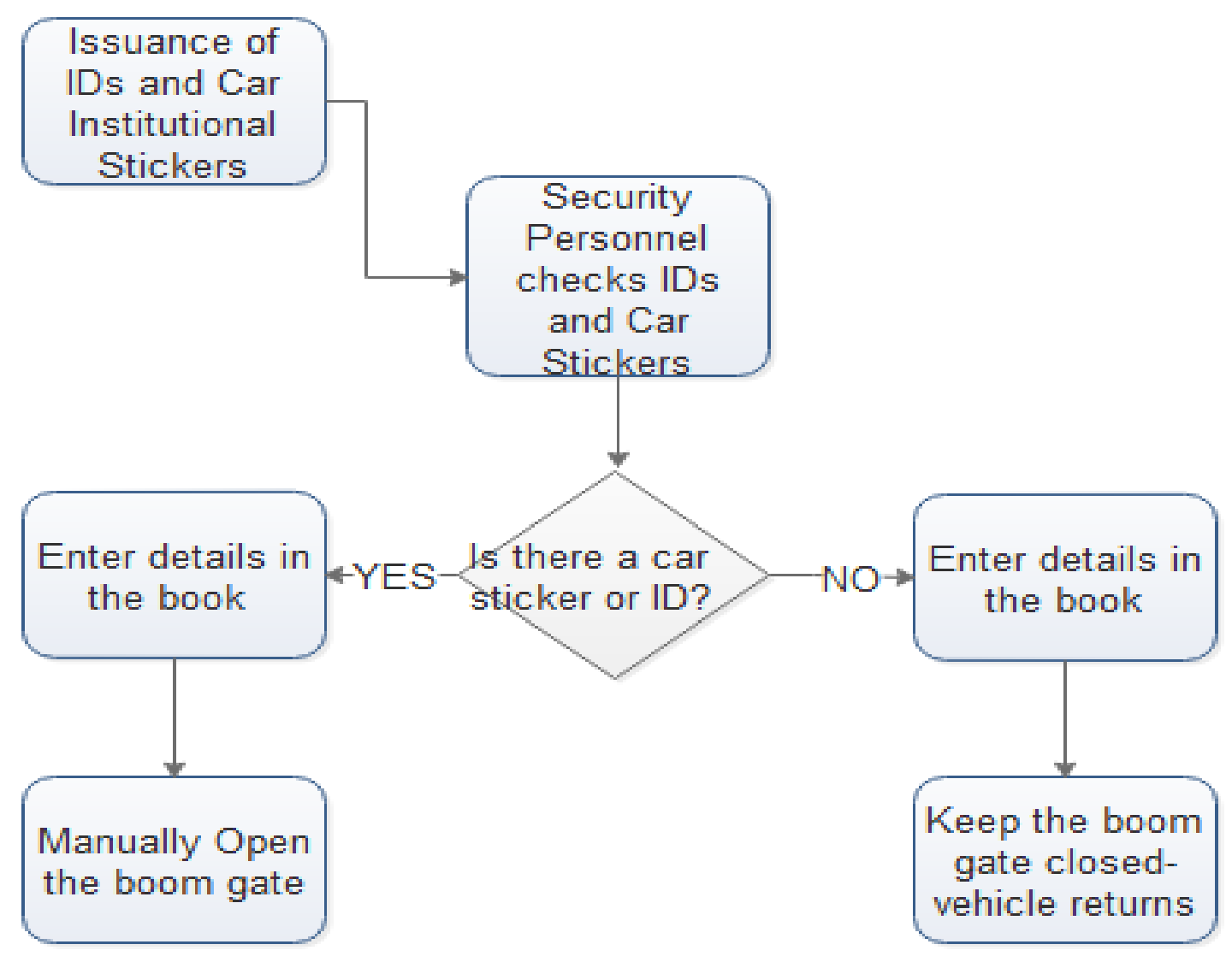

Fig. 4. The Current Manual Vehicle Authentication System. 


\section{- Proposed Vehicle Access Control Model}

In this research study an alternative vehicle Access control model based on automatic number plate recognition and radio frequency identification was proposed. The proposed system automated the current manual system in order to bring efficiency and effectiveness and efficiency in vehicle controls and monitoring. The proposed model is a two factor vehicle access control system using automatic number plate recognition and radio frequency identification. The two technologies were integrated in order to meet requirements at different access points. The proposed system offered five configurable authentication access levels;

1) First Level just takes records of the vehicles entering and leaving through a particular point with direct access.

2) Second is the card OR number plate authentication level, where either of the two would be used to gain access.

3) Third is Membership Identification Card ONLY, where only the identification card was used to gain access.

4) Fourth is the number plate ONLY, where only the car number plate was used to gain access.

5) fifth is the number Plate AND Membership Identification Card

\section{A. Overview of the Proposed Model}

The IR powered cameras were installed beside the road to take pictures of an approaching vehicle. The entry IR sensors when interrupted by an approaching vehicle, triggers the camera to take pictures. The selected picture is then sent for processing in order to extract the number plate in text format. The text number plate is compared to the data in the database and if a match is found, the ANPR system triggers the opening of the boom gate by closing the actuation circuitry switch (Fig. 5). At the same time, the ANPR process is taking place, the RFID reader identifies the driver's membership card (ID). It sends the card's information to the database, if there is a match, the RFID system triggers the opening of the gate to the boom gate by closing the actuation circuitry switch (Fig. 5). If the number in either case does not correspond to the database detail, the corresponding switch remains in the open state, hence the boom gate is kept closed (Fig. 6).

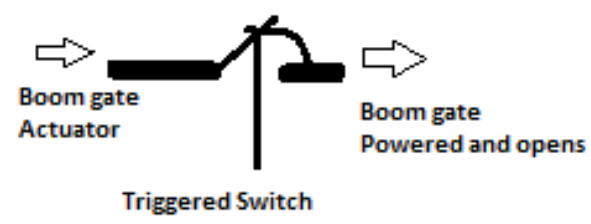

Fig. 5. Triggered Circuitry Switch.

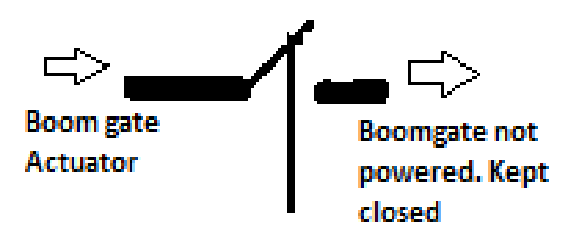

Non trigered switch

Fig. 6. None Triggered Circuitry Switch.

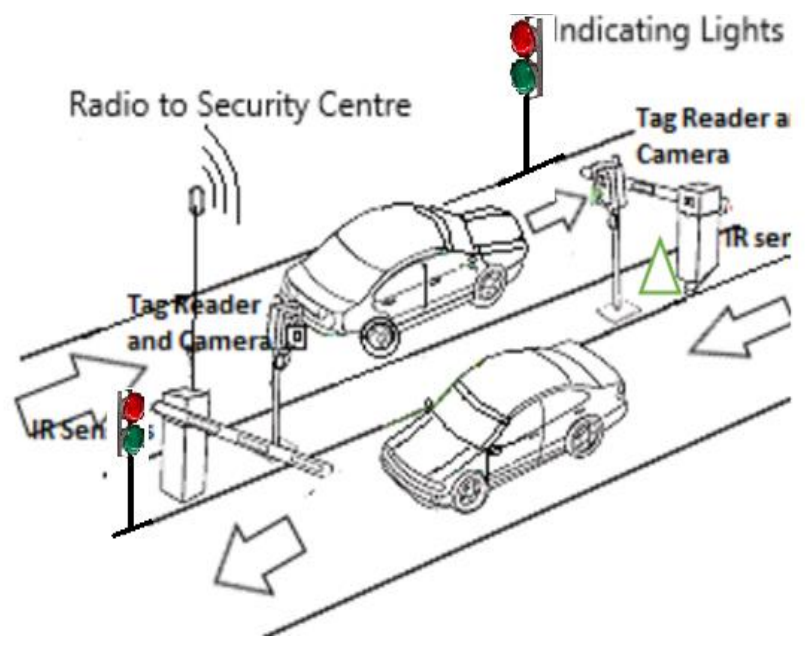

Fig. 7. System Overall View.

The overall system was powered with the boom gates as barriers, Cameras to take pictures, IR sensors to sense the presence of the vehicle, indicating lights giving boom gate status, motor to drive the boom gate, computer, Arduino circuitry and associated components. Fig. 7 displays the overall overview of the system.

- Implementation

Manual methods currently on use for vehicle access control can be replaced with automated system in order to eliminate the challenges experienced by the manual system. In order to show how the two factor authentication system can work at the University of Zambia great east road campus, a prototype was developed to show the proof of concept. The prototype was implemented using RFID and ANPR technology to show how the two systems can be used for a two factor vehicle access control. The development stages included

1) Circuit design and simulation in proteus for RFID and MATLAB for ANPR

2) Device building(on bread board)

3) Circuit board fabrication

4) Building system on circuit board

5) Database design and implementation

\section{B. Automatic Number Plate Recognition (ANPR)}

1) Block Diagram And Flow Chart: From the block diagrams presented in Fig. 8 and 9, as the vehicle is approaching, IR sensors senses the incoming vehicle and activates the cameras to take pictures. The number plate characters are extracted as shown in Fig. 10. The database is queried for the availability of the number plate in the system. After a query response from the database, the microcomputer sends a signal to the boom gate to trigger the switch if the match was verified. When the boom gate opens, it sends a signal to the microcomputer to inform the user that access has been permitted. The microcomputer sends a signal to the display for a message to the user. At the same time exit sensors are activated to instruct the boom gate closure after the vehicle passes. 


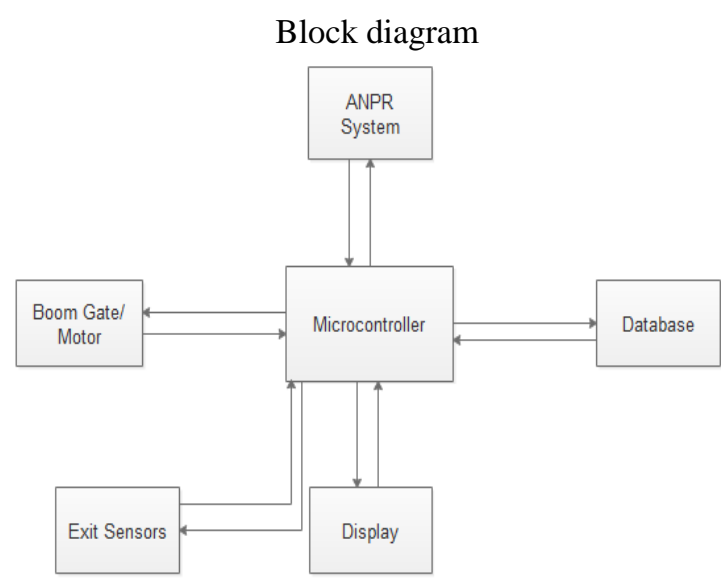

Fig. 8. Block Diagram for the System using ANPR.

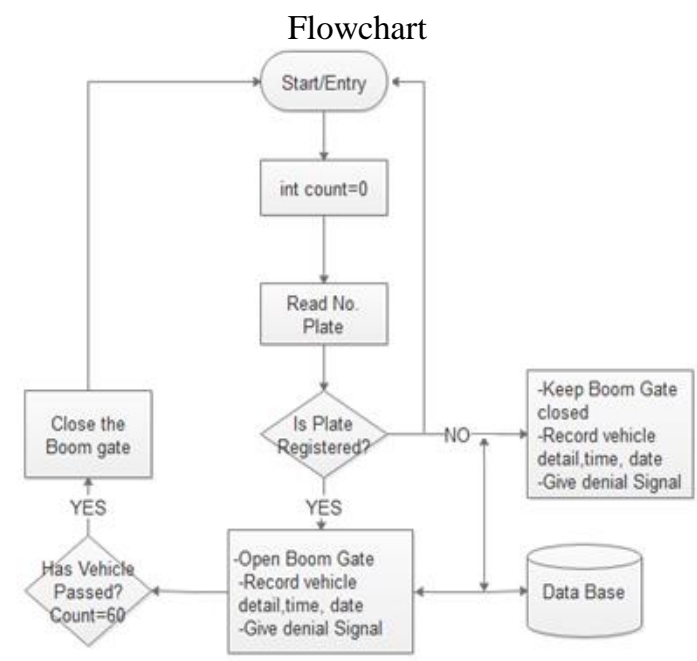

Fig. 9. Flowchart Diagram for the System using ANPR.

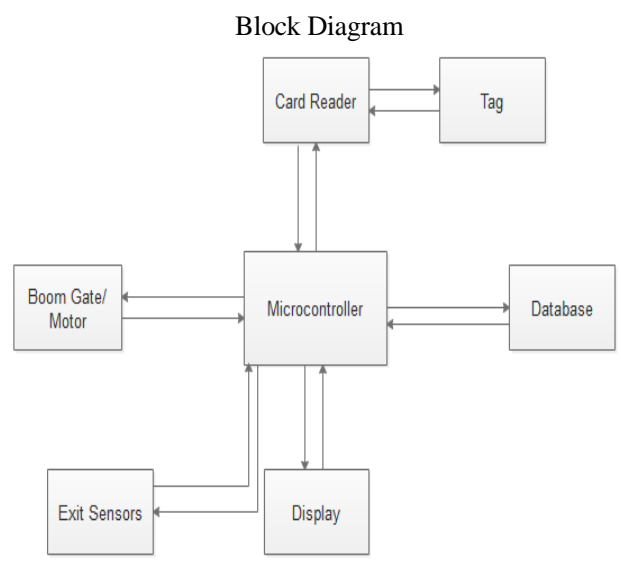

Fig. 10. Block Diagram for RFID System.

After successful extraction of the image in the morphological process, the number plate is converted into its binary form. The final characters are normalized and recognised by the template matching algorithm giving the result shown in the output note text of Fig. 21.

\section{Radio Frequency Identification (RFID)}

1) Block diagram and flowchart: From the diagrams presented in Fig. 11 and 12, the reader reads user information from the tag and sends it to the Microcontroller. The microcontroller queries the database for the availability of similar information. If the query response is positive, the microcomputer sends a signal to triggers the switch to actuate the boom gate or else the switch is kept open. When the boom gate opens, it sends a signal to the microcomputer to inform the user that access has been permitted. The microcomputer sends a signal to the display for a message to the user. At the same time exit sensors are activated to instruct the boom gate closure after the vehicle passes.

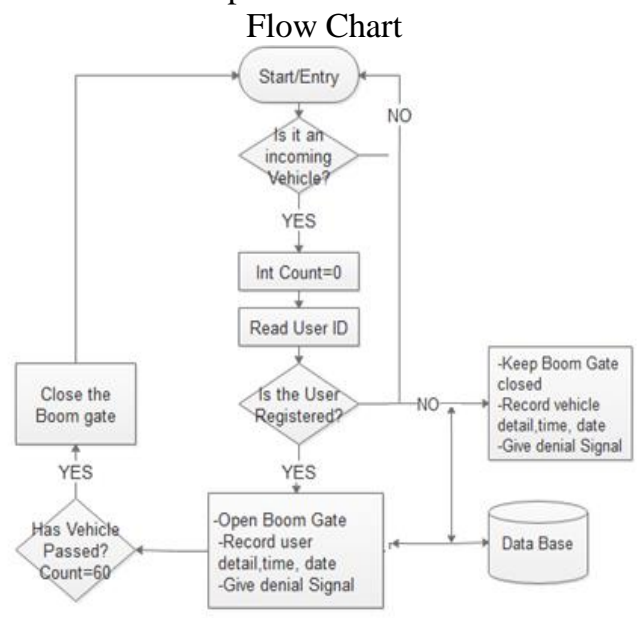

Fig. 11. RFID System Flow Chart.

2) ANPR and RFID integrated system: The two technologies were integrated to offer five access states. The first state provided the recording of vehicle passages while keeping access less restricted.

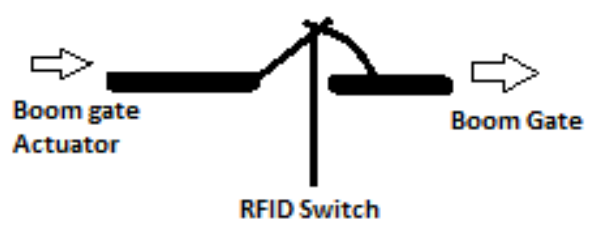

Fig. 12. Switch Actuated by RFID Identification tag ONLY.

The second state was were the only identification required to gain access was the RFID card (Fig. 13). The RFID switch linked the boom gate actuator to the boom gate for opening or closing.

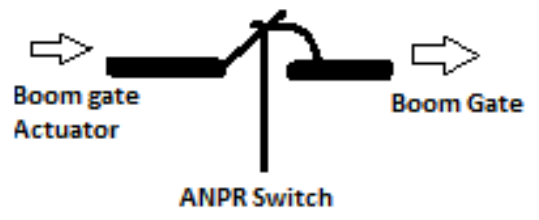

Fig. 13. Switch Actuated by Number Plate Identification Only. 


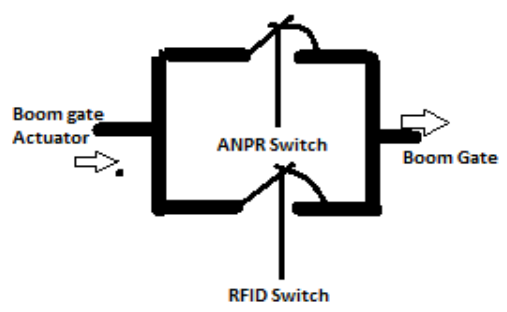

Fig. 14. RFID and ANPR Switches 'ORed'.

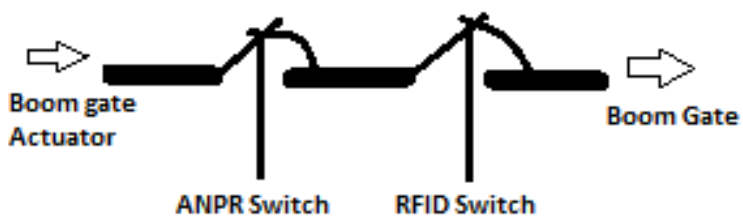

Fig. 15. RFID and ANPR Switches 'ANDed'.

The third state was were the only identification required to gain access was the vehicle number plate (Fig. 13). The ANPR switch linked the boom gate actuator to the boom gate for opening or closing

The fourth state as illustrated in Fig. 14 gave access through either the number plate OR the identification RFID card.

The fifth state was a more secure state that 'ANDed' the two technologies as demonstrated in Fig. 15. It required both the RFID identification card AND the vehicle number plate matched for access to be granted.

\section{RESULTS AND DISCUSSION}

This chapter gives an overview of the system results and an analysis of the results and functionality of the system.

1) Survey: In this section, a sampled results of the baseline study derived from the variable analysis through descriptive statistics are presented. The results are presented in the form of bar charts and pie charts.

Fig. 16 revealed that most respondents understood and were aware that the university had a restrictive policy on vehicles' access into the University premises and car parking areas.

\section{Knowledge on the institutional Policy...}

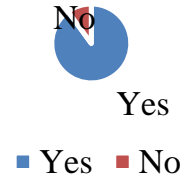

Fig. 16. Knowledge of Institutional Policy on Vehicle Access in the Campus and Car Parks.
Fig. 17 presented the fact that no record is taken on the vehicles that enter the university premises or vehicles that access the car parking areas.

Fig. 18 indicated that the majority of the respondents recommended to have a system that kept records of vehicle activities.

Fig. 19 most staff highly recommended for a secure system that denies vehicle access and exit without the owner's identity cards.

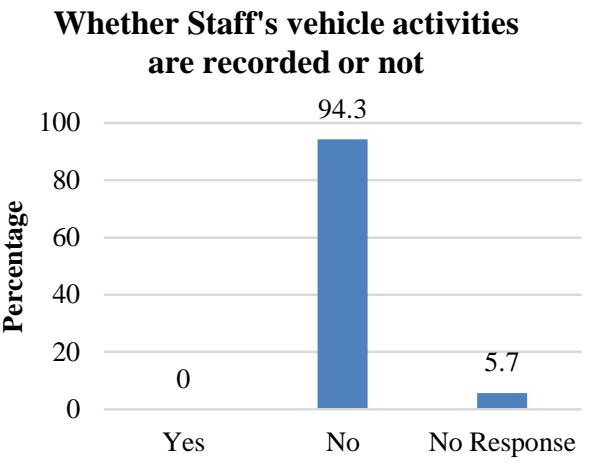

Fig. 17. Recordings of Vehicle Activities in and Out of the Premises.

\section{Recommendations of a sytem to provide vehicle access control and} records

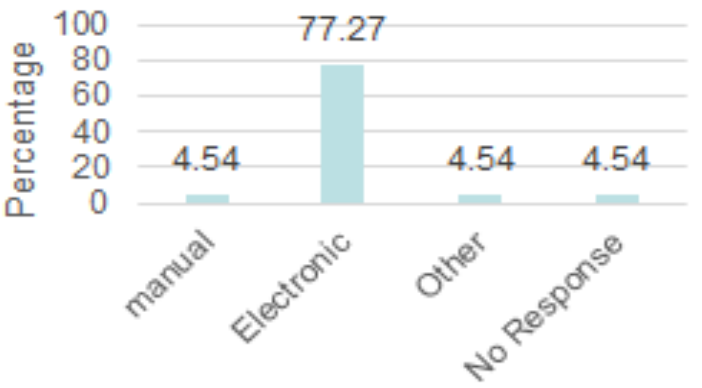

Fig. 18. Respondents Response for Access Control System that Keeps Records.

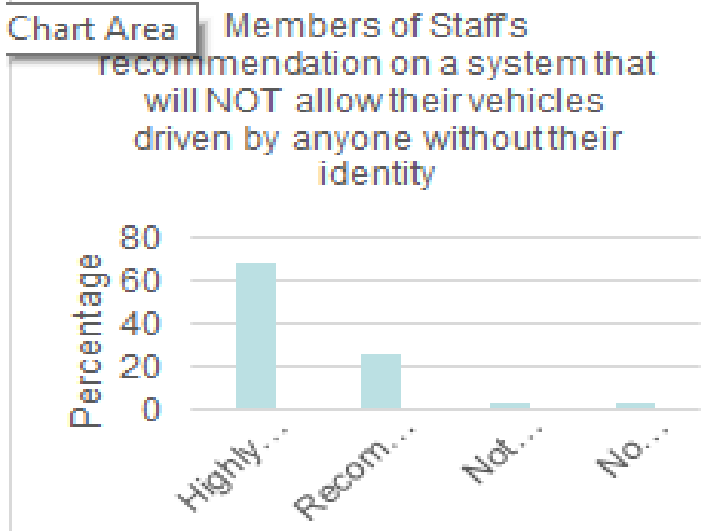

Fig. 19. Access of the Vehicles Without the Owner's Identity Card. 


\section{Knowledge of Car theft from Campus}

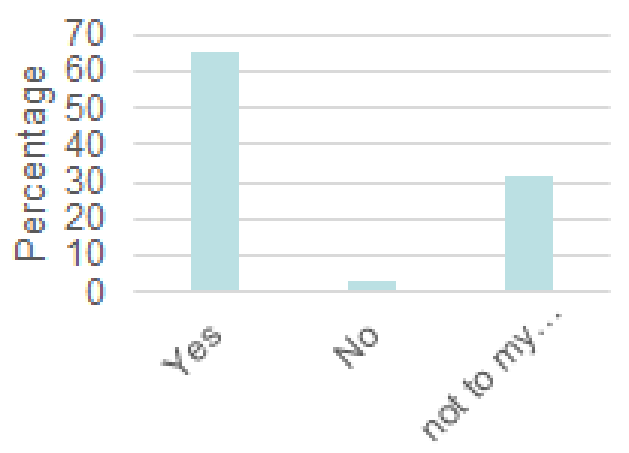

Fig. 20. Respondent's Knowledge Over Car Thefts Within Campus.

Most respondents indicated awareness of vehicle thefts within campus and were not confident on the safety of their vehicles as indicated in Fig. 20.

2) ANPR System (Example of operation): This section gives an example of the process in recognizing the number plate of the system in operation. Fig. 21 shows the process of number extraction from pre-processing stage through to the recognition of the number plate text stage. The system loads an image of a car number plate after camera capture. The image is converted to grayscale and horizontal and vertical edge processing is carried out to extract the edges from the image. Then filtering process is employed. Furthermore the system finds all possible plate regions and highlights them for processing. After successful extraction the image is converted into its binary form. Morphological processes are carried out to ensure unnecessary components are removed. Finally, the characters are normalized and recognised by the template matching algorithm giving the result shown in the output note text as shown in Fig. 21.

3) RFID System (Hardware implementation): The prototype system was constructed, allows card configurations, denies entry to non-programmed cards and allows entry to cards that are programmed.

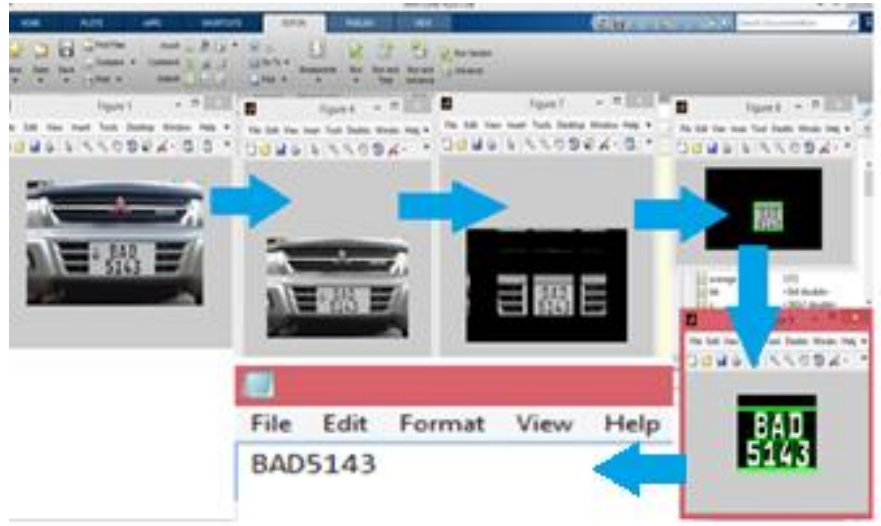

Fig. 21. ANPR Extraction of Number Plate Digits.

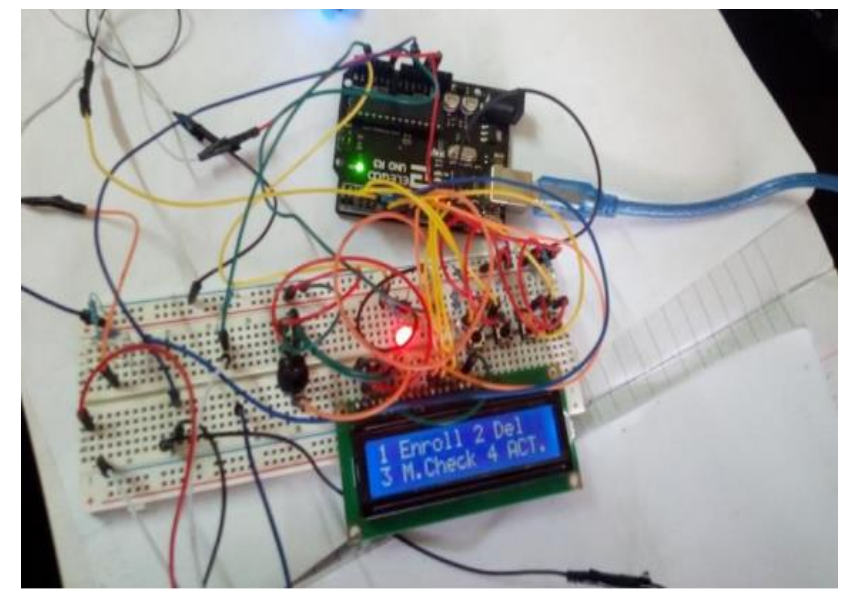

Fig. 22. Prototyping Set-Up at Main Menu.

Fig. 22 shows the main menu from the "Settings" option that allowed systems' administrative configuration of users. The administrator is able to enrol, delete, check or edit users.

When pre-programmed card is introduced to the RFID card reader and the system verifies its authenticity, the system opens the boom gate to allow passage of the vehicle (Fig. 23)

After the vehicle passes passed the exit sensors, the system receives a closing command and the boom gate closes (Fig. 24).

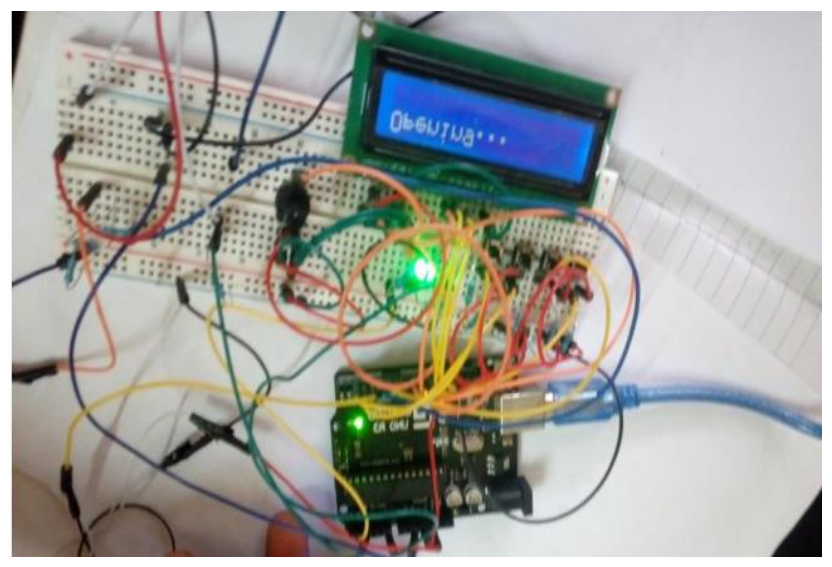

Fig. 23. ID Verified, Open Command Issued.

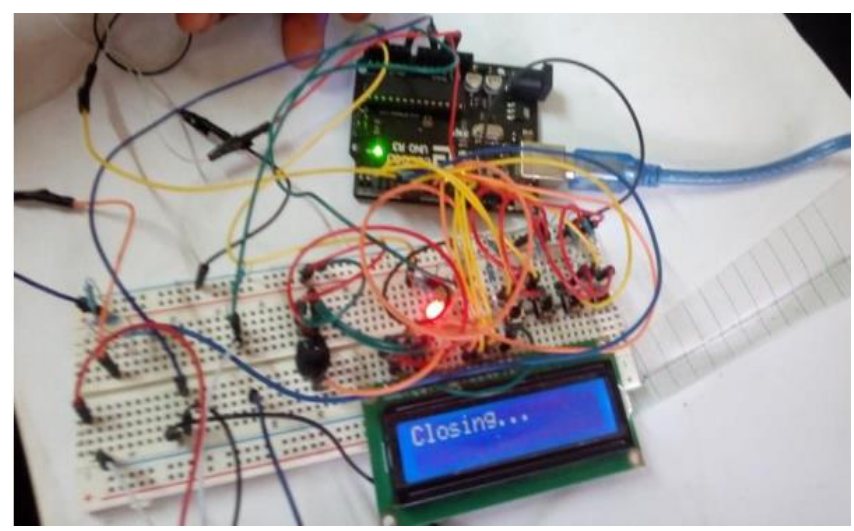

Fig. 24. ID Verified, Close Command Issued. 
4) Data base: The System matches time and date to the access attempts made by the user. It is stored in a 'txt' document created using the application 'Coolterm'. Coolterm is installed and kept running as long as the scanner is operational to properly log the data. The Coolterm application is directly linked to the Arduino's serial port and synchronises with the Arduino's Serial.print and Serial.println functions. Because of this, we are able to extract various amounts of information from the system allowable by the command and with special setting, can attach time and date to the information.

In the example below the Cross Card is enrolled and the other two are not.

\section{Card Information:}

Tick card: 4900DC6F916B

Circle(O) card: 4900DC7032D7

Cross(X) card: 4900CC37DD6F

Fig. 25 shows the txt file called RFID_log.txt and the corresponding Times and Dates of access:

The system is however supplemented by the use of online databases. In this project a possible alternative to this was created using a local server for demonstration purposes using xampp. The database was created using xampp's Apache and MySQL data modules to facilitate data storage. The results are displayed in Fig. 26.

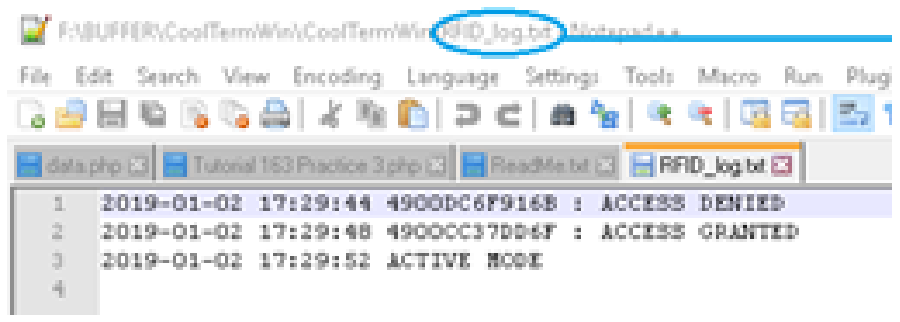

Fig. 25. Database Showing Transaction Activities.

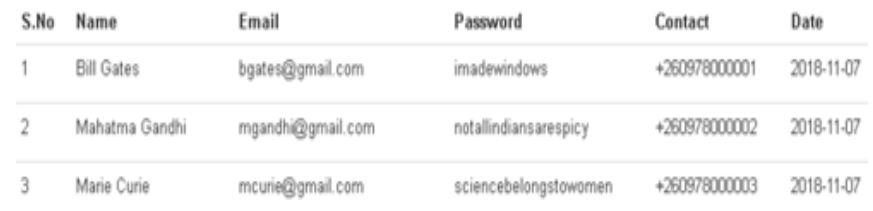

Fig. 26. Online Database Using Apache and MySQL.

\section{RECOMMENDATION}

The following recommendations are forwarded to the University of Zambia for an efficient and effective management of vehicle access controls within and into the University premises.

Automating vehicle controls using RFID and ANPR technologies will bring about efficient and effective vehicle access controls, vehicle monitoring system, tracking vehicle activities in the campus, easier record retrieval mechanism, and prevention of vehicle thefts and preservation of parking space.

The system can further be enhanced to notify the driver on an empty space within the designated car parking area and notify the vehicle owner whenever the vehicle is leaving the premises.

\section{ACKNOWLEDGMENT}

I wish to thank the University of Zambia, Security Department for providing the variable information. I would like to also thank the staff in the Department of Computer Science and School of Engineering for the technical guidance.

\section{REFERENCES}

[1] J. Phiri, T. J. Zhao, and J. I. Agbinya, "Biometrics, device metrics and pseudo metrics in a multifactor authentication with artificial intelligence," IB2COM 2011 - 6th Int. Conf. Broadband Commun. Biomed. Appl. Progr., pp. 157-162, 2011.

[2] J. Phiri, T. J. Zhao, C. H. Zhu, and J. Mbale, "Using artificial intelligence techniques to implement a multifactor authentication system,” Int. J. Comput. Intell. Syst., vol. 4, no. 4, pp. 420-430, 2011.

[3] R. A. N. Karthik, A.K. Parvathy, "Design of Automatic Number Plate Recognition on Android Smartphone Platform - 1," Indones. J. Electr. Eng. Comput. Sci., vol. 5, no. 1, pp. 48-57, 2017.

[4] M. Mohandes, "An Intelligent System for Vehicle Access Control using RFID and ALPR Technologies - 2,” pp. 3521-3522, 2016.

[5] A. Ustundag and M. S. Kilinc, "Design And Development Of RFID Based Automated Car Parking System," vol. 2, no. Figure 1, pp. 6-8.

[6] N. Simin, F. Choong, and C. Mei, "Automatic Car-plate Detection and Recognition System," pp. 113-114, 2013.

[7] K. Sonavane, B. Soni, and U. Majhi, "Survey on Automatic Number Plate Recognition (ANR)," Int. J. Comput. Appl., vol. 125, no. 6, pp. 14, 2015.

[8] S. V. Suresh and T. Sabhanayagam, "Car License Plate Detection using Structured Component Analysis," pp. 196-200, 2014.

[9] A. D. APREM DALAL and P. D. R. B. MAJHI, "Automatic License Plate Recognition System,” Comput. Sci. Eng., vol. BACHELOR, no. Xi, pp. 70-85, 2011.

[10] M. Deepavali and P. A. C. Lomte, "Implementation of an Extensive Number-Plate Recognition \& Authentication (NPRA) System using MATLAB," vol. 3, no. 11, pp. 301-304, 2013. 
[11] P. Rajvanshi, "Automatic Number Plate Recognition-Approch for Detecting the Vehicle Number Plate On-The-Go - 11," Int. J. Comput. Appl., vol. 170, no. National Conference on Cloud Computing \& Big Data, pp. 83-89, 2017.

[12] D. Y. Gaikwad and P. B. Borole, "A Review Paper on Automatic Number Plate Recognition (ANPR) System - 12," Int. J. Innov. Res. Adv. Eng., vol. 1, no. 1, pp. 88-92, 2014.

[13] T. K. \& P. K. Davinder Parkash, "The RFID Technology and ITS Applications: A Review," Int. J. Electron. Commun. Instrum. Eng. Res. Dev., vol. 2, no. 3, pp. 109-120, 2012.

[14] P. M. Senadeera and N. S. Dogan, "Emerging Applications in RFID Technology," Int. J. Comput. Sci. Electron. Eng., vol. 4, no. 2, pp. 7579, 2016.

[15] A. Alexandru, E. Tudora, and O. Bica, "Use of RFID Technology for Identification, Traceability Monitoring and the Checking of Product Authenticity," vol. 4, no. 11, pp. 8-10, 2010.

[16] N. Saxena and A. R. Sadeghi, "a survey paper on radio frequency identification (RFID) trends," Lect. Notes Comput. Sci. (including Subser. Lect. Notes Artif. Intell. Lect. Notes Bioinformatics), vol. 8651, pp. 1-18, 2014.

[17] L. Rajan, A. Gopi, P. R. Divya, and S. Rajan, "A Survey on RFID Based Vehicle Authentication Using A Smart Card," vol. 4, no. 3, pp. 106-110, 2017.

[18] R. Want, "An introduction to RFID technology," IEEE Pervasive Comput., vol. 5, no. 1, pp. 25-33, 2006.

[19] E. Ilie-Zudor, Z. Kemény, P. Egri, and L. Monostori, "The Rfid Technology and Its Current Applications," Isbn, vol. 963, pp. 86586-5, 2006.
[20] R. Das, "RAIN and NFC Market Status, Outlook and Innovations Raghu Rfid 2018-2028:," 2018.

[21] J. Landt, "The history of RFID," IEEE Potentials, vol. 24, no. 4, pp. 8 $11,2005$.

[22] J. Jones, Wyld, \& Totten, "The adoption of RFID technology in the retail supply chain," no. June 2014, 2005.

[23] A. Bhavke, "Advance Automatic Toll Collection \& Vehicle Detection During Collision using RFID Akshay," 2017.

[24] D. L. Almanza-ojeda and M. A. Ibarra-manzano, "Design and implementation of a vehicular access control using RFID - 4," no. December 2006, 2016.

[25] J. Yun and S. S. Lee, "Human movement detection and identification using pyroelectric infrared sensors," Sensors (Switzerland), vol. 14, no. 5, pp. 8057-8081, 2014.

[26] A. Rogalski, "Infrared detectors: an overview," Infrared Phys. \&amp; Technol., vol. 43, no. 3-5, pp. 187-210, 2002

[27] B. Dold, "Infrared Radiation in Modern Technology Infrared Radiation in Modern Technology," no. April, p. 8, 2016.

[28] J. Lofland, "An infrared distance sensor, Analysis and test results," J. Contemp. Ethnogr., vol. 13, no. 1, pp. 7-34, 1984.

[29] B. Izydorczyk, A. Kwapniewska, S. Lizinczyk, and K. SitnikWarchulska, "Infra Red Detectors," Int. J. Environ. Res. Public Health, vol. 15, no. 6, pp. 1-28, 2018.

[30] Jackson Phiri, Tie-Jun Zhao, Jameson Mbale," Identity Attributes Mining, Metrics Composition and Information Fusion Implementation Using Fuzzy Inference System", Journal of Software, Vol 6, No 6 (2011), pp.1025-1033, June 2011 